\title{
Artigos \\ HUELLAS DE UNA INNOVACIÓN METODOLOGICA: "EXPERIENCIAS DEL COMER”, UN PROCESO EN PRODUCCIÓN
}

\author{
Adrian Scribano $^{1}$, Aldana Boragnio ${ }^{2}$, Julia Bertone ${ }^{3}$, Pilar Lava ${ }^{4}$.
}

\begin{abstract}
RESUMEN
La metodología de la investigación social es un campo disciplinar en permanente modificación, en donde la apertura a la creatividad y expresividad en las estrategias de indagación social implican nuevas necesidades. Incluir las vivencias del investigador como rasgo fundamental para mejorar la captación y comprensión del mundo social es uno de los desafíos que se une a inquietudes tales como cómo dar lugar al registro y sistematización de las huellas de un proceso en producción que nos permita mejorar las estrategias de captación y comprensión de las sensibilidades sociales. El objetivo del presente artículo es compartir las vivencias de los investigadores sobre el diseño y aplicación de una experiencia de indagación que implica la puesta en práctica de diversas y articuladas "innovaciones" metodológicas; como la incorporación de la autoetnografía como recurso procesual que brinda miradas necesarias en el proceso de investigación.
\end{abstract}

Palabras clave: auto-etnografía, creatividad, expresividad, sensibilidades, comer.

\begin{abstract}
The methodology of social investigation is a disciplinary field in a constant change, where openness to creativity and expressiveness regarding the social investigation strategies imply new needs. Including the experience of the researcher as one of the main features to apprehend and comprehend the social world is one of the challenges that gives room to record and systematize the footprints of the production process which allow us to improve the strategies of apprehension and comprehension of the social sensibilities. The aim of this article is to share the researcher's experiences on the drawing and application of a research that implies putting into practice several articulated methodological innovations. For instance, the assimilation of autoethnography as a procedural resource that allows a privileged view in the researching process.
\end{abstract}

Keywords: autoethnography, creativity, expressiveness, sensibilities, to eat.

\footnotetext{
${ }^{1}$ Investigador Principal del CONICET, IIGG, UBA / Director del CIES; adrianscribano@gmail.com

${ }^{2}$ Licenciada en Sociología, IIGG, GECE, CIES; aldana_b@ hotmail.com

${ }^{3}$ Licenciada en Sociología, UNVM, GESSYCO, CIES; bertone-julia@ hotmail.com

${ }^{4}$ Licenciada en Sociología, IIGG, GECE, CIES; pilar.lava@gmail.com
} 


\section{Huellas de una innovación metodológica: "experiencias del comer", un proceso en producción}

\section{INTRODUCCIÓN}

La metodología de la investigación social es un campo disciplinar en permanente modificación. Desde el abordaje monográfico de Le Play, pasando por los aportes de W.I Tomas a la indagación cualitativa hasta llegar a las actuales experimentaciones sobre la "investigación basada en el arte" los procedimientos, las prácticas, los objetivos y los fundamentos de las pesquisas científicas sobre la realidad social han sido y siguen siendo objeto de cambios y experimentaciones.

El objetivo del presente artículo es compartir las vivencias en tanto investigadores sobre el diseño y aplicación de una experiencia de indagación que implica la puesta en práctica de diversas y articuladas "innovaciones" metodológicas. La estrategia argumentativa seleccionada ha sido la siguiente: a) se presenta sumariamente las bases conceptuales de la auto-etnografía y del lugar de la creatividad en la investigación social, b) se sintetiza el dispositivo que hemos dado en llamar “experiencias del comer", c) se incluyen las auto-etnografías de tres investigadoras participantes y, d) se concluye abriendo una serie de interrogantes sobre los desafíos que la experiencia plantea.

Se sostiene que la apertura a la creatividad y expresividad en las estrategias de indagación social implican la necesidad de re-incluir las vivencias del investigador como rasgo fundamental para mejorar la captación y comprensión del mundo social.

Uno de los desafíos más importantes que aquí asumimos es dar lugar al registro y sistematización de las huellas de un proceso en producción (sensu Bhaskar) que nos permita mejorar nuestras estrategias de captación y comprensión de las sensibilidades sociales.

\section{AUTOETNOGRAFIA Y EXPRESIVIDAD}

La investigación social, sus estrategias, sus soportes procesuales, sus objetivos cognitivos (y por qué no políticos) son siempre objeto de controversia. Por ello, cuando llevamos adelante un proceso de investigación hay que asumir los límites de éste en 
tanto espejo de la realidad procurando disolver lo que en él haya de mundo social naturalizado.

En este contexto, el lugar del investigador en el proceso de indagación, sus destrezas, sus posición y condición de clase, las tradiciones teóricas y epistemológicas en las que se inscriben adquieren una relevancia particular. Éste es el único que en el proceso de investigación tiene la posibilidad/responsabilidad de ser sujeto y objeto, de gestionar su "ubicación" en la indagación, ya que interactúa con el objeto de estudio a la vez que formula y se formula preguntas y conoce el mundo en tanto sujeto: creencias, emociones, reglas, etc.

La autoetnografía es un recurso procesual de la investigación social que permite captar y "usar" las potencialidades (y obstáculos) que la posición del investigador a la que hemos hecho alusión contiene e implica. En una rápida asociación de ideas, la autoetnografía podría aparecer como una simple "combinación" de autobiografía con etnografía, pero si se profundiza en sus características, vemos que es un recurso en donde al mismo tiempo se involucra la autorreflexión y la emoción.

La auto-etnografía como recurso fue puesto en consideración por algunos autores. Para Smith (2005) la auto-etnografía no es sólo un recurso para la reflexión, sino que aparece como un espacio intermedio entre la pasión y el intelecto, una frontera entre el análisis y la subjetividad. Wall (2006) también se centra en esta técnica como una forma de contactarse con la reflexión del investigador en torno a las preguntas que él elaboró y a sus problemas en el transcurso de la investigación. No es en la reflexión, sino en el papel del investigador como "autor integrado" situado en el centro de la investigación donde Montero-Sieburth (2006) pone el énfasis. En este contexto la autoetnografía puede ser entendida como un recurso que se abre a una exploración de lo emotivo, aproximándose a la reflexión personal y a las interpretaciones intersubjetivas (Scribano y De Sena, 2009), dentro de un proceso de reflexividad que profundice el "papel" del investigador como un sujeto partícipe de los estados de cosas y campo de indagación de la investigación misma.

En ese espacio en donde ambas posiciones del investigador se "encuentran" es donde hay que acercarse e indagar en la expresividad, sabiendo que al "ingresar" a ella aparecen nuevos interrogantes, especialmente en torno a lo teórico y metodológico. A partir de ello aparecen tres problemáticas principales que hay que discutir y tener en cuenta: la primera es aquella que se ubica en torno a las preguntas sobre las estrategias expresivo-vivenciales que demandan una respuesta sobre si las mismas son o no 


\section{Huellas de una innovación metodológica: “experiencias del comer", un proceso en producción}

"técnicas de recolección de datos". La segunda es la que está conectada con los haceres y prácticas del investigador que toma la decisión de trabajar estas vías para captar lo social y la tercera problemática es la cuestión fundamental de cómo registrar 'sentires'. (Scribano, 2014: 7).

La auto-etnografía es un modo de etnográfica que se despliega como estrategia metodológica de investigación cualitativa, que consiste en "aprovechar y hacer valer las 'experiencias' afectivas y cognitivas de quien quiere elaborar conocimiento sobre un aspecto de la realidad" (Scribano y De Sena, 2009: 5), teniendo como punto central la participación del sujeto que investiga como parte del mundo de la vida en el cual está inmerso el aspecto a indagar.

Desde esta perspectiva podemos ver que la auto-etnografía es tanto estrategia cualitativa, es un modo de trabajar con información privilegiada, ya que se re-toma la experiencia del investigador desde su posición excepcional en el campo específico de indagación. Lo que se busca con ello es acentuar su reflexividad y su capacidad de mostrar sus sentimientos/creencias/opiniones con el fin de "utilizar la propia experiencia para ampliar la comprensión de lo social" (Scribano y De Sena, 2009: 6), haciendo foco en las emociones, en el intercambio con los otros, en la relación intersubjetiva que se produce en el momento de "hacer-campo", de experimentar el propio proceso de indagación..

Los sujetos involucrados en el aludido proceso brindan información sumamente substancial para el objeto de estudio, ya sean estos elegidos por su posición específica en relación al problema a trabajar o por ser actores que tienen su vida atravesada por éste. En éste marco la auto-etnografía es pertinente pues el "sujeto-que-indaga" también está incluido en el referido campo problemático: sea porque forma parte de su mundo de la vida o porque es quien lo ha "estado trabajando" lo cual indefectiblemente lleva a que sea parte de dicho mundo. Siendo esta participación/inmersión el motivo por el cual el investigador también posee información específica para brindar.

Scribano y De Sena (2009) conciben a la auto-etnografía como "una estrategia que prioriza y describe la propia experiencia vivida y las variaciones en el modo de otorgarle sentido (...) [en la que] se pone en juego elementos personales y sociales.”; así podemos entenderla como una estrategia en la cual se busca dar cuenta de la propia experiencia en el proceso de investigación en donde se ocupa el lugar privilegiado de ser sujeto y objeto, de ser parte de la estructura global del mundo de la vida del objeto a 
investigar, de estar socializado en la misma cultura que los sujetos a indagar y de ser "arte y parte" del fenómeno que se quiere comprender. Es ésta información privilegiada e irreproducible desde otro lugar en el proceso, información fundamental que se perdería sino es incorporada como estrategia de indagación.

Con esta perspectiva de base, no hay que perder de vista algunos rasgos de las Ciencias Sociales que tienen que ser puestos en cuestionamiento y reformulación. Entre ellos encontramos que las definiciones epistémicas de la conexión entre percepción, observación y conocimiento científico deben ser tenidas en cuenta para poder trabajar en su "ampliación"; al mismo tiempo que las relaciones entre la forma de expresar el sentido de la acción y los procesos que llevan a esa expresividad deben ser pensados a través de una reconceptualización basada en la apropiación científica de medios tecnológicos y artísticos para observar lo social (Scribano, 2014a). Por lo que, si estamos interesados en indagar sobre las sensibilidades, hay que pensar en nuevas estrategias que nos permitan acercarnos, y de una nueva forma, a la expresividad de los sujetos y la creatividad.

En esta estrategia el conocimiento y la experiencia se ponen en acción, incentivando que el investigador se acerque a las preguntas que el mismo elaboró, a la reflexión de su problema de investigación y de la comprensión de su experiencia como parte de lo social a comprender (Scribano y De Sena, 2009), pero desde un lugar diferente, desde su participación como sujeto y desde sus propias emociones. Dicho de otro modo, haciendo foco en el proceso dinámico de la propia experiencia. Desde la perspectiva tomada, se sostiene que el carácter dialéctico que tienen las dimensiones metodológicas, teóricas y epistemológicas se pone claramente en juego "cuando queremos indagar las sensibilidades, ya que cuando uno parte de la encarnación de los cuerpos en las geometrías corporales y en las gramáticas de las acciones" (Scribano, 2014: 3) aparece el desafío de construir un modo de indagación a partir de las sensibilidades.

La Sociología de los Cuerpos/Emociones aparece como el terreno predilecto de estas cuestiones, en donde se vuelve indispensable la creatividad por parte del investigador al poner en juego la dialéctica de las Ciencias Sociales, ya que lo que la determina -la triada teoría-epistemología-metodología- se une a la premisa que no se puede diferenciar las emociones del cuerpo.

El estudio de las emociones no es algo nuevo. Desde épocas pasadas podemos ver un interés en las Ciencias Sociales por buscar en las emociones especificidades que 


\section{Huellas de una innovación metodológica: “experiencias del comer", un proceso en producción}

se escapan en los relatos de las estrategias tradicionales anteriores. Por eso, si queremos indagar sobre las emociones es indispensable adentrarse en el pasado para comprender las preguntas y problemas que surgieron, que pueden ser parte de nuestras preguntas actuales y pensar los modos de darles respuestas ya que "aquellas estrategias nos sirven para pensar creativamente la metodología hacia el futuro" (Scribano, 2014: 2).

Pensando al cuerpo y a las emociones en su dialéctica constitutiva, vemos que lo importante del proceso es que el investigador pueda lograr que éstas afloren. Pero ese florecer, ese aparecer, no se da en términos de un "desocultamiento", de un descubrimiento. Las emociones no se encuentran en el fondo mismo del sujeto y esperan a que éste las saque al exterior, sino que son constitutivas y constituidas en el momento de la expresión de estas. En este contexto de discusión, es donde nos preguntamos ¿qué dicen sobre lo social y lo individual los cuerpos? ¿Qué dicen sobre lo social/individual esos cuerpos que se expresan, esas sensibilidades? El cuerpo aparece como el espacio de articulación de "unas respuestas que juegan en el aquí/ahora, antes/después y se establecen como borde respecto a la palabra" (Scribano, 2014a: 5).

En tanto la vida de los sujetos refiere a la experiencia de la tensión entre sociabilidad-vivencialidad-sensibilidad, aparece la posibilidad de elaborar un conocimiento a través de los cuerpos (Scribano, 2014); pero seguida a esta posibilidad, surge la duda sobre cómo rupturar los dispositivos de regulación de las sensaciones. Por ello el investigador debe centrarse en uno de los papeles más importantes, lograr las condiciones necesarias para que los sujetos se expresen -y al mismo tiempo, él se exprese.

El expresarse suele aparecer asociado al crear, y éste, mayoritariamente al arte. Pero por fuera de esta asociación el crear es parte intrínseca de todo ser humano, se relaciona con el expresarse, con realizar un acto afirmativo de la autonomía en el cual el sujeto expone sus sensibilidades (Scribano, 2014).

Si esas sensibilidades son la expresión del sujeto, de su creación, entonces podemos decir que expresarse es realizar un acto de habla que da cuenta de la relación entre el cuerpo y la emoción, en donde ambos están constituidos por esa relación. Como dijimos antes, hay una vinculación -una tensión- entre el sujeto, su cuerpo, sus emociones y lo social y esa vinculación se genera a través del sujeto en el mundo. Dicho de otro modo, el sujeto y lo social se vinculan en las experiencias que tenga el sujeto, que puede expresar a través de la creación como acto afirmativo de su autonomía. Así lo 
que el sujeto expresa será un indicio de esa vinculación, pero un indicio que se relacione con las experiencias de ese sujeto; como algo vinculado con la historia que el sujeto va construyendo con su cuerpo, una vinculación consigo mismo y con lo social. (Scribano, 2014)

Repitamos, el papel del investigador al indagar estas aristas de la experiencias se centra no sólo en su atención, empatía y en el desarrollo de sus preguntas de investigación, sino en crear las condiciones necesarias para que el sujeto se pueda expresar. Lo que buscamos es que los “objetos" devenidos sujetos de indagación, puedan expresarme mediante su expresividad y al mismo tiempo, que el investigador encuentre su espacio de expresión propia, como sujeto participante de la experiencia de indagación.

En este expresarse del sujeto podemos ver los "estados de sensibilidad que, como sujeto y sociedad, [se han] ido tramando y constituyendo" (Scribano, 2014: 10) Se trata de crear una experiencia para que el sujeto tenga una experiencia y en ello se centra la metodología basada en la expresividad, "el sujeto dispone de la imaginación que se ha ido construyendo colectiva y socialmente; nosotros, como metodólogos, creamos las condiciones para una experiencia de las experiencias (Scribano, 2014: 9).

En lo que sigue reseñamos el diseño y aplicación de lo que hemos dado en llamar "experiencias del comer" en tanto dispositivo que intenta captar sensibilidades.

\section{EXPERIENCIAS DEL COMER: DISEÑO Y CONTENIDOS}

En la búsqueda de caminos alternativos para investigar las conexiones entre sensibilidades, expresividad y conocimiento de la sociedad diseñamos un dispositivo que hemos dado en llamar Experiencias del Comer. El objetivo es indagar algunas de las prácticas del sentir que están asociadas a la comida. En ese contexto, se explora a) la relación comida, color y sensaciones, b) las conexiones entre color y experiencias del comer anteriores, c) el lugar de lo visual en los sabores, y d) cuales son los nexos existentes entre comida y sociedad.

Dispositivo: La experiencia está dividida en tres momentos:

1. Primer momento: en una mesa se colocan seis platos blancos con tarjetas de colores (blanco, negro, amarillo, verde, negro, rojo y naranja). Se le solicita a los 


\section{Huellas de una innovación metodológica: "experiencias del comer", un proceso en producción}

sujetos participantes que elijan una tarjeta según un color y un sabor al que lo asocian. Se le pide a cada uno de los participantes que cuenten el motivo por el cual eligieron ese color y se les pregunta: ¿Qué esperan comer habiendo elegido dicho color?

2. Segundo momento: se les tapan los ojos a todos los partícipes, y posteriormente se les da, a cada uno, el plato de comida (acorde al color seleccionado). Blanco: rodaja de pan cubierta de queso Brie y pera natural, Negro: mousse de chocolate, Verde: niños envueltos, Naranja: pincho de langostinos y gajos de naranja, Amarillo: polenta cocida al horno con huevo duro y Rojo: tarta de frutillas. Luego de que prueben la comida, se les pregunta: ¿qué comieron? Y ¿qué relación tiene con lo que esperaban comer?

3. Tercer momento: se les destapan los ojos a todos los participantes, y (con el plato sobre la mesa) se inicia el diálogo para que cada uno narre la experiencia experimentada.

Los sujetos participantes se seleccionan, mediante la red de contactos del equipo de trabajo, seis personas de 30 años en adelante, contemplando la diversidad sexual y la variedad etaria de los participantes.

La experiencia es filmada con dos cámaras hogareñas que permiten tener una mirada más compleja sobre la experiencia facilitando el registro y posterior análisis de la misma.

Durante el año 2013 se concretó una experiencia donde participaron las siguientes persona cuyas características se exponen a continuación según el color seleccionado por ellos: Amarrillo: mujer, 34 años, terciario completo, universitario en curso y trabaja en el estado; rojo: varón, 31 años, estudios de posgrado, trabaja en análisis de mercado; naranja, mujer, 42 años, nivel de estudios terciario, trabaja en diseño y comercialización de objetos de decoración, blanco: varón, 29 años, nivel de estudios terciario, trabaja en comercialización (venta) de alimentos; negro: varón, 33 años, nivel estudios universitario incompleto, trabaja en el estado como curador de arte; y verde: mujer 29 años, nivel de estudios universitario incompleto, trabaja en atención al público en local comercial de Palermo.

Algunas notas sobre la experiencia aludida pueden ser sintetizadas del siguiente modo: 
NORUS - v1, n.2, jan-jun 2014.

\section{Primer momento:}

La persona que seleccionó el amarrillo manifestó "me gusta mucho melón y el ananá, sobre todo melón...si como helado pido de melón” Mientras la que escogió el naranja dijo “...por la sed, tengo sed y pienso en algo color naranja...si no hubiera elegido el blanco también...”

\section{Segundo Momento:}

Con los ojos tapados comienzan a narrar e interrogados sobre lo que esperaban comer y la experiencia que están teniendo comienzan a expresar lo siguiente:

"Tarta de frutillas, que es uno de mis postres favoritos ...esperaba encontrar algo salado...(¿qué sentís?, pregunta del coordinador)...placer”' (Comensal que eligió el rojo) .

\section{Tercer momento:}

Ya con los ojos destapados el coordinador consulto sobre la valides de un dicho popular que dice "La comida entra por los ojos" y todos asintieron: "Si la comida entra por los ojos ..."

En la trama de los tres momentos y de manera provisional es posible sostener que:

Se pudo advertir una relación directa entre comida, colores e biografía. Cuando los participantes refieren a qué asocian el color de lo que están de comiendo emerge fuertemente los recuerdos familiares.

Los participantes asocian lo multicolor con lo rico, con lo sabroso, con lo divertido y atrayente, conectan directamente experiencia estética y experiencia del comer. 


\section{Huellas de una innovación metodológica: “experiencias del comer", un proceso en producción}

Se puede observar que entre las expectativas de la comida de acuerdo al color seleccionado y lo degustado efectivamente existe un alto grado de disonancia si bien se acepta que lo comido se asocia al color escogido.

Los participantes explicitan claramente que las conexiones que ellos realizan entre colores y sabores son tributarias de experiencias del comer anteriores que han marcado sus gustos personales.

Fue posible advertir que los participantes asocian los colores de lo "natural", cuyo paradigma son las ensaladas, con lo atractivo y gustoso para comer.

Es en este contexto que las vivencias como investigadores en el curso y transcurso de la experiencia esquematizada devienen tanto un eje central de los que se "vivió-entre-todos" como un "recurso hermenéutico" fundamental para seguir explorando en y a través de la creatividad.

Es en el contexto aludido donde hemos querido incorporar la auto-etnografía como una estrategia para mejorar y cualificar nuestra experiencia de indagación sobre las sensibilidades.

\section{AUTOETNOGRAFIAS: COMO SE VIVE UNA INNOVACIÓN METODOLOGICA}

Las auto-etnografías que presentamos a continuación se escribieron con la intensión de registrar ¿Qué pasaba con las sensibilidades de aquellas que estaban tratando de captar las experiencias de otros? Muy "rudimentariamente" el explicitado objetivo buscaba re-capturar a posteriori lo que se había vivido para transformarlo en recurso de indagación por un lado y "conservarlo" como huella de una experimentación metodológica.

En el orden que aparecen, los presentes escritos fueron producidos por Aldana Boragnio, 28 años, socióloga (UBA) iniciando su proceso de formación de maestría en Investigación Social (UBA) y participante del Grupo de Estudio de Cuerpos y Emociones (GECE) - Instituto Investigación Gino Germani (UBA). Julia Bertone, 30 años, profesional gastronómica, socióloga (UNVM), doctoranda en Ciencias Sociales (UBA-CONICET), miembro del Programa de Estudios de Acción Colectiva y Conflicto 
NORUS - v1, n.2, jan-jun 2014.

Social (CIES-CONICET). Y María del Pilar Lava, 32 años, socióloga (UBA), maestrando en Diseño y gestión de Programas Sociales (FLACSO, Argentina) Miembro del GECE y del GEPSE5 . Participa en Palermo Intenso, proyecto de investigación sobre la estructura de las sensibilidades del circuito de diseño y gastronomía del barrio de Palermo de la Ciudad de Bs As.

\subsection{Los colores de la comida, la comida de colores}

Desde el momento que planteamos el encuentro de experiencia de comensalidad como una actividad a desarrollar no supe bien qué iba a pasar. La idea me parecía interesante y que podía dar lugar a cosas nuevas, aunque no sabía bien a qué. Invitar a un grupo de 6 personas a comer. Invitar a un grupo de 6 personas a comer a ciegas. Las dudas eran varias; conseguiríamos rápidamente personas dispuestas a participar, seguirían sus ganas de formar parte cuando les digamos que se comía a ciegas, que no sabíamos cuáles iban a ser los platos.

Que alimentos y de qué forma íbamos a organizar los platos fue un tema de debate; sólo un alimento, varios alimentos pero ordenados por preponderancia de color en el plato, por mismo color en el plato. La última fue la elegida y se hicieron seis platos distintos en dónde los ingredientes -todos o mayoritariamente- del plato reflejaban el color elegido. Ahora, ¿salado o dulce? ¿Algo agridulce? La decisión fue mezclar, dos platos dulces, tres salados, y uno agridulce. Que sean tibios o fríos fue más una cuestión de comodidad y posibilidades físicas de la cocina.

Conseguir participantes dispuestos no resultó nada complicado, completamente diferente a lo que había pensado. Sólo nos quedaban cuestiones de organización práctica y división de tareas. Mi rol fue filmar el encuentro, principalmente debía buscar un ángulo amplio para lograr un plano general de la mesa, teniendo en cuenta que todos entren en la imagen. Desde ese momento mi atención se basó en que la luz roja de la cámara esté prendida; primero porque no me manejo fluidamente con los aparatos tecnológicos y segundo porque el momento era ese, si llegaba a pasar algo que hiciera que no se esté filmando, el momento era irrepetible.

La gente fue llegando y tímidamente se ubicaron alrededor de la mesa al tiempo que iban charlando unos con otros. La mesa estaba lista, la gente ansiosa y los aromas

\footnotetext{
${ }^{5}$ Grupo de Estudios sobre Políticas Sociales y Emociones ( GEPSE) del Centro de Investigación y Estudios Sociológicos (CIES).
} 


\section{Huellas de una innovación metodológica: “experiencias del comer", un proceso en producción}

invadían el espacio, dando pie a preguntar qué pensaban que habían cocinado. Al momento de iniciar la experiencia, cada uno en sus respectivos lugares, todos miraban las tarjetas de colores ya sabiendo cuál sería la elegida -aunque después descubrimos que faltaba saber el por qué. Cuando fue el momento, rápidamente cada uno tomó una tarjeta y tres hombres se disputaron entre el rojo, blanco y el negro. Uno se quedó con el blanco sin saber bien el motivo.

En el momento de explicitar el porqué de la elección, qué pensaban que comerían, empezaron a surgir las respuestas que estábamos esperando sin saberlo. En su totalidad -con excepción del hombre de blanco- la elección se debió a lo que era su comida preferida o lo que gustaban comer. Verde, por lo fresco, lo liviano, las ensaladas. Rojo por las frutillas, "amo la torta de frutillas" dijeron. Amarillo por el melón, el ananá, “de chica comía mucho melón y ahora siempre como ananá”. Negro por experiencias pasadas, por haber sido siempre un gran momento cuando comía fideos negros.

Era el momento de las vendas. Sutil, pero claramente, se detuvo el tiempo. Se generó un silencio que denotaba la atención, ansiedad y dudas de lo que vendría.

Llegaron los platos, el aire se llenaba de aromas varios, no eran distinguibles, pero era algo conocido. La "cocinera” le detalló a cada uno cómo comer lo que tenían en frente, "es para comer con la mano, está tibio", “acá tenés el tenedor, pinchá por acá", "esto hay que cortarlo, pero se come con cuchara". Con dificultades, con la lentitud y cautela de no tener disponible el sentido más desarrollado de la modernidad, cada uno se acercó a su plato. La mayoría, primero lo olió, buscando una familiaridad, algo que los acerque a la respuesta de lo que vendría. Yo estaba expectante, intentando tomar con la cámara las caras, los gestos de cada uno, qué sucedía con esos movimientos musculares difíciles de controlar que se generan ante lo nuevo, esa respuesta inmediata de los sentidos.

Lo primero que sucedió fue que la mujer que eligió el color verde inmediatamente supo de qué se trataba, "son niños envueltos, la comida de mi casa". Así, con los ojos todavía tapados, cada uno fue diciendo qué era la comida, qué sentían, qué les pasaba. Los hacía recordar a su infancia, a su casa; era la comida que más le gustaba; no se lo esperaba por completo ya que estaban seguros que era algo salado y les tocó una torta de chocolate. Tres de los invitados no supieron qué era lo que comían. El hombre que eligió el blanco, perdido por su elección desde el primer momento, ahora 
estaba perdido en los sabores, “es queso, no sé cuál, pero es queso. Pero es queso y algo más”. La mujer que eligió amarillo no podía saber qué era, aunque le resultaba familiar, había una cremosidad que la desconcertaba. Y la mujer que eligió naranja no supo. Ella, al no saber entró en una ansiedad notoria por no darse cuenta de qué era. Era carne, pero no era cerdo porque no le gustaba, no era pollo. Parecía pollo, pero no era pollo.

Al sacarse las vendas y ver los alimentos sucedieron cosas sorprendentes, sobre todo con quienes no sabían qué habían comido. La mujer que tenía el color amarillo no se había dado cuenta de que era polenta porque, ahora sabía, la había desorientado el huevo poché. La chica "naranja" al ver los langostinos se sorprendió. No sabía cómo se llamaban pero sabía que no le gustaban; o más interesante aún, no sabía si le gustaban porque nunca los había comido pero, viéndolos, nunca lo hubiese hecho.

Luego del núcleo de la actividad el ambiente se distendió mucho más, la gente comenzó a hablar entre sí y sueltamente contaban qué les había pasado. Uno de los ejes más importantes de la charla surgió cuando se preguntó por qué gustaba algo; la infancia y los primeros sabores fueron la respuesta contundente. La familia, el entorno, la historia propia de cada uno, tiene que ver con lo que comemos, con lo que nos gusta. Así los niños envueltos parecen llevar a alguien directamente a su casa, a su patio, a ese patio lleno de plantas que tiene una pared verde y que envuelve la vida en familia al igual que la hoja de parra envuelve al arroz condimentado. Otro tema que surgió y resulta interesante para volver a escucharlo y seguir charlando es la influencia de los colores en los alimentos. No sólo es algo a tener en cuenta a la hora de elegir una comida sino que la combinación de varios de ellos produce un plato colorido y "divertido". Así, se dijo que hay cosas que si tuvieran otros colores no darían ganas de comer; el ejemplo que surgió fue el de las salchichas, que al ser grises parecen podridas. En ese momento yo estaba compenetrada en la actividad de filmar, pero sin parar de escuchar la charla que me generaba unas ganas de participar que debía reprimir. La conversación fue terminando a la vez que variaba entre los gustos, los colores y las combinaciones para ir centrándose en cómo comemos lo que tenemos a mano y que podríamos comer otras cosas.

Al momento de ordenar y lavar todos los utensilios, de limpiar y guardar todos los elementos, me invadió la sensación de haber finalizado algo una actividad atractiva; una actividad que me pareció salió muy bien, muy fluida y muy dinámica, muy conversada y muy vivida, pero que al mismo tiempo ya me generaba ganas de repetir. Tener que repetir la actividad fue lo primero que me surgió. Tenía en claro que se 


\section{Huellas de una innovación metodológica: “experiencias del comer", un proceso en producción}

habían dicho cosas interesantes, cosas que valían la pena reproducir y analizar, al mismo tiempo que las ganas de repetir y buscar conexiones y rupturas con otras personas, otras clases sociales, otras dinámicas, me generó inmediatas expectativas.

Al otro día llamé a mi amiga que había participado -y eligió el color amarillo-, quería saber qué le había parecido, qué pensó. Primero me agradeció por invitarla y me comentó que no sólo se sintió muy cómoda y que le pareció una buena experiencia personal, sino que pudo pensar sobre la comida desde los comentarios de los otros participantes, a la vez que me dijo que nunca había pensado que los colores pudieran estar tan íntimamente relacionados a la comida y al gusto propio.

La experiencia, en su totalidad, me pareció una actividad interesante, nueva, prometedora, y que puede brindarnos información sobre las experiencias del comer a través de las dimensiones de las sensaciones, sentimientos y emociones particulares de los comensales. Que al ser compartida con otras personas en la misma situación se da la posibilidad de conversar más fluidamente, llegando a temáticas que no se habían pensado o que no se habían podido poner en palabras en la soledad del pensamiento momentáneo que aparece al elegir un plato.

Como herramienta metodológica, considero que mediante estas experiencias se consiguen datos y dinámicas de participación que nos permiten pensar y analizar situaciones tan disímiles a otras como puede ser el momento en que el sujeto, con la relación y variedad de comida de todos los días, ocupa la posición de comensal.

\subsection{Color, Cuerpo y Emociones}

\section{LA PREVIA}

La actividad: Hombres $(\mathrm{H})$ y mujeres $(\mathrm{M})$ eligen un color y asocian una comida a dicha tonalidad, con los ojos vendados se les ofrece a cada comensal una degustación con el objetivo de dialogar sobre lo que esperaban comer, lo que identifican que están probando a ciegas, y las emociones y recuerdos ante cada bocado.

Soy cocinera, por tanto dentro del grupo de estudio compuesto por Adrián Scribano, Aldana Boragnio y Pilar Lava, mi tarea fue la de decidir y preparar la comida, que habíamos acordado debía ser simple. Era el lugar más cómodo y felíz que podía tener ya que sintetizaba mis dos intereses, la sociología y la cocina. Era diciembre, la cocina del lugar donde realizaríamos la actividad no contaba con heladera, ni horno, 
NORUS - v1, n.2, jan-jun 2014.

sólo podía usar hornallas en caso de tener que saltear o recalentar en el momento, por tanto las preparaciones debían estar avanzadas y tenían que trasladarse.

Lo que aparentaba ser una tarea sencilla me despertaba dudas en cada alternativa posible ¿Qué color prevalece en un plato de comida? ¿Un color determina el sabor dulce, salado o agridulce de un alimento; o da la pauta de la temperatura fría, tibia o caliente de una receta? ¿La carne vacuna es roja, marrón o gris? ¿El color lo establecería el producto principal de la receta o me guiaría por la presentación final? El rojo del tomate se vuelve tenue cuando se licua, la manzana o pera es blanca si se les retira la piel.

Cocinar en función de un color no era una novedad, consulté un gráfico "cocinando y saboreando emociones" del libro Los postres de Jordi Roca del restaurant El Celler de Can Roca (Roca, 2011: 25) que marca un paralelo entre estados de ánimo (melancolía, ternura, femeneidad, alegría, euforía, masculinidad, agresividad) y estados sensitivos (caliente, seco, áspero, amargo, dulce, suave, húmedo, frío) provocado por diversos sabores (vainilla, nueces, geranio, enebro, miel, jazmín, batata, bergamota) y colores (tierra, leche, rosa, limón, menta, cuero, pimienta); también me dejé llevar por las fotografías de las revistas de cocina que tenía a mano y conté la experiencia que estábamos ideando a mi pareja y amigos quienes se mostraron entusiastas con la experiencia.

Ante la dificultad de comer con los ojos vendados pensé en ofrecer a los comensales tarteletas individuales y variar el relleno según el color: verde/acelga; amarillo/choclo; naranja/zanahoria, etc. Sin embargo ésta posibilidad resultaba acotada ya que todos comerían tarta. Por lo que decidí ofrecer opciones variadas de sabor y temperatura, así cada participante podía dar cuenta de su particularidad.

\begin{tabular}{|l|l|l|l|}
\hline COLOR & PREPARACION & $\begin{array}{l}\text { INGREDIENTE } \\
\text { QUE REFUERZA }\end{array}$ & COMENTARIOS \\
\hline NEGRO & $\begin{array}{l}\text { Mousse de } \\
\text { chocolate /Frio }\end{array}$ & $\begin{array}{l}\text { Pimienta negra } \\
\text { molida }\end{array}$ & $\begin{array}{l}\text { La porción se compra en el } \\
\text { momento, resulta más } \\
\text { económico que hacerlo. } \\
\text { Con cuchara. }\end{array}$ \\
\hline BLANCO & $\begin{array}{l}\text { Queso brie con } \\
\text { pera sobre rodaja }\end{array}$ & Almendra pelada & $\begin{array}{l}\text { El queso brie puede ser } \\
\text { reemplazado por cualquier }\end{array}$ \\
\hline
\end{tabular}


Huellas de una innovación metodológica: “experiencias del comer", un proceso en producción

\begin{tabular}{|c|c|c|c|}
\hline & $\begin{array}{l}\text { de pan casero / } \\
\text { Tibio }\end{array}$ & & $\begin{array}{l}\text { queso cremoso ya que } \\
\text { resulta un sabor más } \\
\text { familiar. Se toma con la } \\
\text { mano. }\end{array}$ \\
\hline VERDE & $\begin{array}{l}\text { Niños envueltos en } \\
\text { hoja de parra / } \\
\text { Caliente }\end{array}$ & Menta fresca & $\begin{array}{l}\text { Es una preparación de } \\
\text { procedencias árabes y } \\
\text { armeña. Las hojas de parra } \\
\text { no son habituales en la } \\
\text { dieta familiar porteña. } \\
\text { Se sirve en cuenco y se } \\
\text { toma con la mano. }\end{array}$ \\
\hline ROJO & $\begin{array}{l}\text { Tarta de frutilla / } \\
\text { Frio }\end{array}$ & $\begin{array}{l}\text { Pétalos de geranio } \\
\text { rojo }\end{array}$ & $\begin{array}{l}\text { Con altas temperaturas se } \\
\text { evita poner crema, se arma } \\
\text { en el momento: masa } \\
\text { quebrada + mermelada y } \\
\text { fruta. } \\
\text { Se toma con la mano. }\end{array}$ \\
\hline AMARILLO & $\begin{array}{l}\text { Huevo poche sobre } \\
\text { polenta grillada / } \\
\text { Caliente }\end{array}$ & Sal de limón & $\begin{array}{l}\text { El huevo no debe reposar, } \\
\text { ideal porcionar en bocados. } \\
\text { Con tenedor. }\end{array}$ \\
\hline NARANJA & $\begin{array}{l}\text { Brochette de } \\
\text { naranja } \quad y \\
\text { langostinos } \\
\text { grillados / Tibio }\end{array}$ & Curry & $\begin{array}{l}\text { En la degustación las } \\
\text { naranjas se soltaron de la } \\
\text { brochette y quedaron solo } \\
\text { langostinos. Revisar si } \\
\text { hacer ensalada (con } \\
\text { tenedor) o brochette (con la } \\
\text { mano) sin naranja a vivo y } \\
\text { solo con alineo. }\end{array}$ \\
\hline
\end{tabular}

Fuente: Tabla elaborado por los autores.

\section{$\underline{\text { EL EVENTO }}$}

Cuando llegaron todos los invitados Adrián Scribano presentó al equipo y dio comienzo a la primera actividad. Mientras los participantes elegían un color y 
comentaban lo que esperaban comer yo me encontraba en la cocina emplatando las preparaciones frías, salteando langostinos, recalentando polenta y niños envueltos. El margen de tiempo fue acotado ya que los participantes resolvieron sin dificultad la tarea y dieron su exposición de manera fluida. Mis compañeras ayudaron a vendar a los participantes y alcanzamos los platos a la mesa. Las dificultades que surgieron fueron resueltas en el momento: pérdida de vendas negras, descorche de vino apresurado, dificultad técnica con una de las filmadoras; cuestiones que de repetirse la experiencia se pueden evitar y tienen que ver con el detrás de bambalinas.

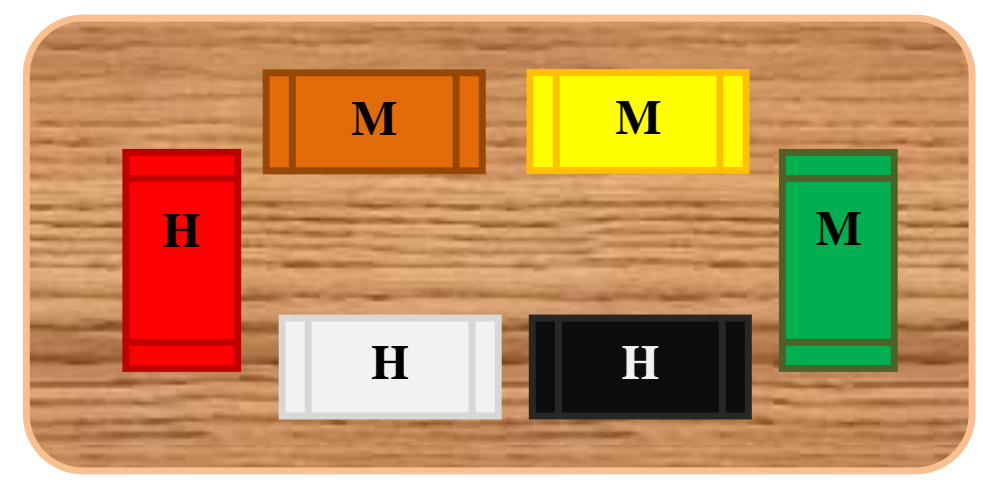

Fuente: La figura fue preparada por los autores.

A cada comensal le fuimos dando indicaciones de cómo tomar su preparación, en algunos casos con la mano, otros con cuchara o tenedor; y en simultáneo se fueron dando las diferentes reacciones ante el primer bocado.

La menta fue reconocida espontáneamente y se olfateo como un ramo de flores, así los niños envueltos empezaron a saborearse uno a uno, después la comensal aclaró que su familia era de procedencia árabe y ésa era una receta habitual que le recordaba a su infancia y a un patio de paredes verde. El primero en romper el silencio expresó "estoy comiendo frutillas" y coincidía con las expectativas que tenía al elegir el color rojo. El brochette de langostinos resultó indescifrable, era la primera vez que la comensal probaba esa carne dulce y de extraño perfume. Después contó que no comía mariscos porque pensaba que no le gustaban. El que esperaba salado recibió sorpresivamente el dulce de la mousse de chocolate; la polenta fue para la comensal un sabor suave y conocido pero no habitual, esperaba choclo pero no encontraba los granos al saborear y eligió el amarillo porque su fruta preferida era el melón. Como responsable de los sabores en juego me sorprendió asociar el melón al amarillo pero quedó claro que fijar un color con un producto resulta dificultoso y generalmente 


\section{Huellas de una innovación metodológica: “experiencias del comer", un proceso en producción}

responde no a un color fijo sino a una gama de colores que, en el caso del melón puede comprender tonalidades de naranja y amarillo pasando por el verde, asociación que dependerá del lugar y el tiempo histórico de quien prueba, su biografía y experiencia con dicha fruta. El comensal que optó por el blanco no podía discernir con claridad qué probaba, pero los sabores le hacían recordar a un plato que le gustaba mucho de chico y le cocinaba su mamá: matambre al roquefort con manzanas; y la asociación resultó lógica, a ciegas había probado queso brie con pera sobre rodaja de pan casero, en ambos platos estaba lo agridulce, el queso y la fruta.

Cuando los participantes se retiraron las vendas, ya con la vista en juego empezaron a probar y compartir las diferentes preparaciones. Mi lugar había pasado de cocinera a observadora. Noté que la comensalidad y experiencia común doto al grupo de un lazo que, aunque artificial y breve, provocó un diálogo fluido. Así, el condicionamiento económico y las diferencias socioculturales en la elección de la comida, las tradiciones y herencia familiar, la moda que dota de estatus a ciertos alimentos como la entraña (corte vacuno que con el tiempo aumentó su valor) o la rúcula, la rutina alimenticia y la necesidad de incorporar ciertos alimentos fueron algunos temas que se hablaron entre los comensales.

Emoción, placer, recuerdos de la infancia, desconcierto, aprendizaje y alegría fueron alguna de las sensaciones que salieron en juego ante la experiencia de degustación a ciegas.

Quienes habíamos estado organizando el encuentro nos sumamos a una picada general donde las recetas se pusieron en juego.

Algo que fue sugerido al equipo es consultar por alergias o restricciones de ingerir algún alimento a próximos participantes de actividades como éstas.

\section{DESPUÉS EN CASA}

Finalizado el encuentro resultó necesario repetir la experiencia de modo que se sedimenten y confirmen las primeras intuiciones de trabajo: que el color como disparador de comidas particulares es una matriz que se aprende socialmente, en relación con otros; que la alimentación compartida e intercambiada actúa como signo de cohesión, y la reciprocidad del acto de saborear implicó la palabra. La boca se evidenció como instancia fronteriza entre el afuera y el adentro de quien prueba. La predisposición sensorial testimonia la capacidad para disponer la interioridad, manifestar un juicio 
sobre las cosas, y evocar recuerdos de infancia, identidades y lugares. Repensé las ventajas y desventajas de hacer la experiencia a ciegas. Creo que profundiza la necesidad de saborear, tocar y olfatear la comida en tiempos en donde todo entra por los ojos. Me sorprendió que quien esperaba dulce recibió salado y viceversa, pero ningún comensal se sintió defraudado sino que la sorpresa y el cambio de sabores resultó novedoso y enriquecedor para todos. Además me reconfortó la confianza que depositaron los invitados en la confección de las preparaciones y las expresiones de satisfacción al probar la comida.

\subsection{Autoetnografía de la Experiencia de Comensalidad}

Para iniciar este relato considero pertinente contarles que justamente al momento de escribir mi auto etnografía sobre la experiencia colectiva que habíamos realizado, me encontré con una dificultad. En vez de contar mi experiencia personal, mis pensamientos y emociones, contaba los de los otros, es decir objetivaba la experiencia describiendo los que hacían los participantes. De modo tal que, en vez de escribir una auto etnografía redacté las notas de campo de un observador participante.

Para salir de esta situación, y romper con esta inclinación me pregunté: ¿cuál era mi relación con la comida? E inmediatamente me retrotraje a mi infancia, recordé una foto de pequeña en la cual comía, justamente experimentando la textura de la comida. Pero creo que lo que me trajo a la mente esa imagen es una particularidad, mi mamá me ponía un pintorcito (delantal plástico) para que no me manche la ropa en el momento de la comida. No me limitaba el experimentar porque mi cara y pelos se veían embadurnados de comida, peor si le ponía cierto tinte de "higiene/ pulcritud" al ritual.

Luego retomando la pregunta, otra vez pensé en mi mamá, no había duda de que mi primer referente en relación a la comida era ella. Mi mamá después de mi año de vida se hizo macrobiótica, si bien yo comía variado y otro menú distinto al de ella, prefería las pasas de uva antes que las golosinas.

Mi padre también interviene en mi relación con la comida, a él le gustaba salir a comer a restaurantes con mucha frecuencia. En esas reiteradas salidas, mi plato elegido era el mismo, nunca pedía otra cosa, siempre comía ñoquis con salsa de crema. Ahora, a la luz de la experiencia lo de comensalidad colectiva que hicimos, no puedo evitar asociarlo con los colores. Mi plato preferido, de chica, era cien por ciento blanco! Color 


\section{Huellas de una innovación metodológica: “experiencias del comer", un proceso en producción}

que hoy no elegiría de ningún modo para un plato de comida, me resulta insípido y asociado a cuando uno se siente mal.

Ya de adulta en mi formación como socióloga, la comida también estuvo presente, en varios períodos de la carrera trabajé en el ámbito gastronómico. La comida, la comensalidad; el placer culinario; la gastronomía en general y el contrastante Mundo de los No culinarios se fue transformando en un objeto exploratorio de indagación sui generis para mí. La curiosidad por comprender ciertos comportamientos y relaciones con la comida me llevó a tener preguntas recurrentes, y el intentar dar respuestas tentativas al lugar ocupado por la práctica del comer en nuestra sociedad. Particularmente en los espacios de la ciudad de ciudad de Buenos Aires que frecuentaba. Finalmente el circuito gastronómico del barrio de Palermo, motorizó mi curiosidad hasta transformarse en un tema de indagación sociológica para mí.

Luego de lo escrito, me siento en condiciones de empezar a redactar sobre mi experiencia en el marco de la experiencia colectiva.

Como decía, dos párrafos arriba, trabajé en el ámbito gastronómico, lo que dio una familiaridad con el hecho de ver gente degustando comida, opinando y hablando sobre ello.

Por lo que en la previa a la realización de la experiencia no me generaba una gran incertidumbre, que los invitados puedan hablar sobre los platos que iban a saborear. Lo que si me preocupaba era la ejecución "correcta" de los pasos de la Experiencia de Comensalidad que habíamos diseñado colectivamente, en otras palabras me preocupaba más los que podía pasarnos a nosotros como equipo de trabajo, más que lo que podría pasarle a los invitados con los estímulos de la experiencia.

Ahora, al escribir sobre mis sensaciones en los momentos previos a realizar la experiencia me doy cuenta de que confiaba en el dispositivo que habíamos diseñado. Las incertidumbres que tenía estaban relacionadas con los aspectos más operativos del dispositivo, no con el diseño del mismo.

El día pactado para realizar el evento aludido, los miembros del equipo nos encontramos horas antes de que lleguen los participantes en el CIES $^{6}$. La previa fue bastantes calurosas, era uno de esos primeros días sofocantes de verano, en los que no corre una gota de aire, y en los que todavía el cuerpo no está acostumbrado a sentir tanto

\footnotetext{
${ }^{6}$ Centro de Investigación y Estudios Sociológicos (CIES).
} 
NORUS - v1, n.2, jan-jun 2014.

calor. Tal vez exagero y no hacia tanto calor, pero para mí preparar lo planeado fue a un ritmo lento bajo los efectos de esta temperatura sobre mi cuerpo.

Habíamos dividido tareas, cada uno llevaba y preparaba algo distinto, nos encontramos con algunos inconvenientes, por ejemplo no teníamos mantel (no lo habíamos coordinado bien entre nosotras), no conseguimos hielo para mantener la temperatura de las bebidos, pero pese a eso detalles pudimos cumplir con lo necesario para recibir a los participantes con el dispositivo preparado. Igualmente estaba un poco nerviosa tenían que llegar todos los invitados.

Los invitados fueron referenciados por los miembros del equipo, en función de los criterios (cuota de edad, lugar de residencia y género) que se habían acordado en el momento del diseño del dispositivo. A algunos de estos invitados los había contactado yo, era mi responsabilidad su presencia en el CIES. La cita era a las 19 hs, a medida que fueron llegando les ofrecimos bebidas que tomando mientras íbamos "rompiendo el hielo" entre todos y esperábamos al resto de los participantes. Una de las invitadas que yo había contactado estaba retrasada, había tenido un inconveniente en el subte y no llegaba, eso me puso nervios. Sumado a que otro de los invitados era el amigo, de una amiga, que había confirmado su presencia, pero hasta que no llegó no me quedé tranquila. Finalmente una vez que los seis participantes estaban presentes en el CIES iniciamos la experiencia.

Adrián Scribano, coordinó la experiencia e inició la actividad en primer lugar presentando al grupo y luego explicando a los participantes cual era el motivo de la convocatoria. Para mí fue muy clara y amena la introducción que él dio, pero tenía la duda de si para los invitados (que no eran sociólogos) también era claro lo que él comentaba.

Desde mi percepción el ambiente que se generó entre los invitados y el equipo fue agradable, todos estábamos expectantes. Nosotros, como equipo, solo contábamos con los pasos del dispositivo que habíamos diseñado, dado que esta era la primer experiencia de comensalidad que hacíamos. Los participantes no tenían mucha noción de que iban hacer, en el momento que los invitamos solo le contamos lo básico, por lo cual su presencia en el CIES ya anticipaba su predisponían y ganas de experimentar algo nuevo.

Luego de la presentación, el primer paso fue invitar a los seis participantes a la mesa y solicitarles que elijan una tarjeta según el color del que desean que sea su comida. 


\section{Huellas de una innovación metodológica: “experiencias del comer", un proceso en producción}

Para mí, en el momento de la experiencia, era difícil ser miembro de equipo y observador a la vez, estaba atenta a lo que tenía que hacer, en ese momento que los invitados elegían las tarjetas de colores, yo estaba buscando las vendas para tabicar los ojos que íbamos a entregarles una vez que todos tengan su tarjeta seleccionada. Mientras las buscaba, veo que las tarjetas con colores más vivos (rojo, amarillo, naranja y verde) fueron las primera en ser seleccionadas, en cambio la de color negro y la de color blanco quedaron como última opción. Los dos participantes hombres que tuvieron que repartírselas se miraron des animados, parecía que no las querían, es decir no tenían muchas ganas de comer algo color blanco o color negro. Ahora mientras redacto mi auto etnografía, no puedo evitar asociar que para ellos el blanco (como para mí, ahora de adulta) era un color de comida poco seductor, a diferencia de lo que me pasaba en mi infancia, que era la tonalidad de mi plato preferido. En esta "coincidencia" creo que hay una pista para luego explora entre los el color blanco, la comida y las sensaciones.

Ahora bien, una vez ya seleccionadas las tarjetas estábamos en condiciones de realizar el segundo paso. Les entregué las vendas a los participantes para que ellos mismos se tabicaran los ojos, pero por error una de las participantes tomo dos vendos, entonces, hasta que nos dimos cuenta de eso, pensábamos que nos faltaba una, es decir uno de los invitados no podía tabicarse los ojo. Lo cual para mí era imposible, porque las había contado y cortados horas antes, y las había guardado a las seis vendas juntas. Obviamente, me puse nerviosa ¡nuevamente! Empecé a buscarlas sin saber por donde, hasta que finalmente la participante se dio cuenta que tenía dos vendas juntas. Ya resuelto el inconveniente estábamos listos para el próximo paso.

Se les fue entregando la comida a los participantes para que degusten los platos correspondientes según el color que cada una había elegido. Para ese entonces yo estaba filmando con una cámara de fotos la experiencia, dado que por unos momentos la filmadora que estábamos usando dejó de funcionar.

El momento de ver saborear la comida a los participantes fue agradable, ellos exploraban sus platos con la curiosidad que yo lo hacía de chica. El hecho de no ver la comida los colocó en otro lugar. Mientras ellos descubrían que estaban comiendo, yo los filmaba con la cámara, estaba ubicada en el costado izquierdo de la mesa, por lo cual tenía más proximidad con tres de los participantes. Sus experiencias son las que más tengo presente, y constituyen mis recuerdos más fuertes de esos momentos. 
NORUS - v1, n.2, jan-jun 2014.

Después de un tiempo prudencial en el que todos entablaron una relación con su comida, es decir o ya sabían que estaban comiendo o ya se sentían totalmente desconcertados y sin más recursos para descifrar cual era su plato, iniciamos la ronda en la que los participantes contaban sus experiencias.

El primer participante en describir su experiencia, fue el comensal de tarjeta roja, sus palabras fueron gratificantes para mí. Contó que la tarta de frutilla que estaba comiendo era justamente lo que esperaba comer, y en lo que había pensado en el momento que eligió la tarjeta, dado que asociaba el color rojo con algo dulce. Una de las primeras palabras para que uso para describir lo que sintió al degustar la comida fue placer. Para mí fue una palabra clave, automáticamente me relaje, y perdí la sensación de tensión que había acumulado. Sentí que las categorías analíticas utilizadas en el marco académico empezaban a salir de la voz de los participantes. Para mí en ese momento la experiencia comenzaba a ser un éxito, estaba contenta.

Fuera del marco del CIES, días posteriores, conversando con la participante de tarjeta naranja ella me comentó que había disfrutado la experiencia, sobre todo el hecho de desnaturalizar la práctica del comer. Me contó que el comer es algo que realiza sin reflexionar al respeto y que la experiencia le dio esa posibilidad hasta el punto de que tuvo que pensar como fue construyendo en su historia de vida que alimentos le gustaban y cuáles no.

Repeticiones, contradicciones, pistas para avanzar, moralejas, en definitiva: experiencias. Estas vivencias sobre el experimentar nos dejan en condiciones de extraer algunas reflexiones orientadas al principal objetivo del presente trabajo.

\section{CONCLUSIONES}

Hacer una vivencia, construir una experiencia, destituir los prejuicios y doxas, inscribirse en los intersticios de la corrección académica no es una tarea fácil: demanda una vigilancia epistemológica permanente.

Estas auto-etnografías que hemos compartido con el lector casi "a mano alzada", recién escritas, sin muchos filtros quieren establecer una primera apertura encaminada a repensar desde nuestras vigilancias epistémicas nuestras propias prácticas.

Estas auto-etnográficas nos permiten, al compartirlas, pensar sobre nuestras prácticas: como sujetos interesados en el comer como juego metonímico para pintar las sensibilidades sociales, como participantes de una vivencia que queremos registrar, 


\section{Huellas de una innovación metodológica: “experiencias del comer", un proceso en producción}

recordar y re-comprender y como seres humanos que al compartir con otros sus vivencias comprenden mejor sus haceres.

En primer lugar, el esfuerzo de la autoetnografia y la dificultad de mirarse como un "mero" observador participante. Re-tomar la acción desde nuestras propias sensibilidades no es una tarea para la cual estemos formados, exige un punto de inflexión donde la vivencia adquiere todo su carácter cognitivo. Lo cual nos desafía a pensar en una practica especifica como la que terminamos de narrar, sobre los "espacios" que dejan abiertos/cerrados la dialéctica entre experimentar/conocer/reproducir. Es decir, al construir una experiencia debemos ser capaces de "registrar" nuestra vivencia en primera persona.

En segundo lugar, los obstáculos del hacer adquieren sentido en la necesidad de repetir la experiencia, que si bien será otra, marcará lo que de ella queremos aprender. Experimentar metodológicamente implica re-hacer desde las propias prácticas con el objetivo de profundizar y mejorar el "procedimiento" como una oportunidad para conocer desde nuestra propias posiciones en él.

En tercer lugar, los criterios de validez de las experimentaciones metodológicas se anudan a lo que intersubjetivamente podamos valorar de ellas. La mirada de aquellos para los cuales "creamos" la experiencia juega el rol de validación externa mas acá de la palabra y se ancla en una vivencia común. La recuperación del ellos/nosotros a través del "lo que vivimos todos" es una pista, no definitiva, pero fundante de la validez de la experiencia.

Por estas tres vías concurrentes podemos sostener, al menos de modo provisorio, que experimentar se liga directamente con el construir, registrar y comprender sensibilidades, lo cual nos deja nuevamente en camino para mejorar nuestra capacidad para entender lo que hay de social en ellas.

\section{BIBLIOGRAFÍA}

MONTERO-SIEBURTH, M. (2006). La auto etnografía como una estrategia para la transformación de la homogeneidad a favor de la diversidad individual en la escuela. http://www.uned.es/congreso-inter-educacion-intercultural/Grupo_discusion_1/74.pdf

ROCA, J. (2011). Los postres de Jordi Roca. Más de 80 dulces recetas concebidas en el Celler de Can Roca. Oceano Ambar. Barcelona, España. 
SCRIBANO, A. (2014). "Interludio. Indagando sensibilidades: aproximaciones metodológicas desde la expresividad y la creatividad" en Magallanes, G.; Gandia, C. y Vergara, G. (Comp.) Expresividad, creatividad y disfrute. Editorial Universitas, Córdoba. Argentina. En prensa

SCRIBANO, A. (2014) "Entrevista Bailada: Narración de una travesía inconclusa" en Intersticios Vol 8, N², Madrid, España. Segundo semestre.

SCRIBANO, A Y DE SENA, A. (2009). Construcción de conocimiento en Latinoamérica: Algunas reflexiones desde la auto-etnografía como estrategia de investigación. Cinta Moebio 34:1-15. Disponible en: http://www2.facso.uchile.cl/publicaciones/moebio/34/scribano.html

SCRIBANO, A. Y D'HERS, V. (2013) La performance como herramienta de indagación. VII JEMC- Séptimas Jornadas sobre Etnografía y Métodos Cualitativos. Buenos Aires. mls

SMITH, C (2005). Epistemological intimacy: A move to autoethnography. International Journal of Qualitative Methods 4(2), Article 6. http://www.ualberta.ca/ iiqm/backissues/4_2/pdf/smith.pdf

WALL, S. (2006). An autoethnography on learning about autoethnography. International Journal of Qualitative Methods 5(2), Article 9. http://www.ualberta.ca/ iiqm/backissues/5_2/pdf/wall.pdf.

Recebido: 26/03/2014.

Aprovado: 20/05/2014. 